European

Thyroid Journal
Eur Thyroid J 2014;3:217-226

DOI: $10.1159 / 000367968$
Received: July 4, 2014

Accepted after revision: August 28, 2014

Published online: December 6, 2014

\title{
Platelet-Derived Growth Factor: A Key Factor in the Pathogenesis of Graves' Ophthalmopathy and Potential Target for Treatment
}

\author{
Sita Virakul ${ }^{a, b} \quad$ Leendert van Steensel $^{a} \quad$ Virgil A.S.H. Dalm ${ }^{a}$ Dion Paridaens ${ }^{c, d}$ \\ P. Martin van Hagen ${ }^{\mathrm{a}-\mathrm{c}}$ Willem A. Dik ${ }^{\mathrm{a}}$ \\ Departments of ${ }^{\mathrm{a}}$ Immunology and ${ }^{\mathrm{b}}$ Internal Medicine, Erasmus MC, University Medical Center Rotterdam, and \\ ${ }^{c}$ Rotterdam Eye Hospital, Rotterdam, The Netherlands; ${ }^{d}$ Department of Ophthalmology, Geneva University \\ Hospitals, Geneva, Switzerland
}

\section{Key Words}

Graves' ophthalmopathy · Platelet-derived growth factor . Orbital fibroblast · Proliferation · Hyaluronan - Cytokines . Thyroid stimulating hormone receptor $\cdot$ Dasatinib · Imatinib mesylate $\cdot$ Nilotinib

\begin{abstract}
Activation of orbital fibroblasts resulting in excessive proliferation, cytokine and hyaluronan production and differentiation into adipocytes, is a main determinant of orbital tissue inflammation and tissue expansion in Graves' ophthalmopathy (GO). During the last years we have shown that the platelet-derived growth factor (PDGF) isoforms PDGF-AA, PDGF-AB and PDGF-BB are increased in orbital tissue from $G O$ patients with active and inactive disease. These PDGF isoforms exhibit the capacity to stimulate proliferation, hyaluronan and cytokine/chemokine production by orbital fibroblasts. Moreover, PDGF-AB and PDGF-BB increase thyroid stimulating hormone receptor (TSHR) expression by orbital fibroblasts, which enhances the orbital fibroblast activating capacity of the THSR stimulatory autoantibodies present in Graves' disease (GD) patients. Of these PDGF isoforms PDGF-BB exhibits the strongest orbital fibroblast activating effects, which is likely related to its ability to bind both the PDGF-receptor (PDGF-R) $a$ and PDGF-R $\beta$ chains. Thus the PDGF-system fulfills important roles in orbital fibro-
\end{abstract}

blast activation in both active and inactive GO, which supports a therapeutic rationale for blocking PDGF signaling in GO. Tyrosine kinase inhibitors (TKIs) may be candidates to target PDGF signaling. Of several TKIs tested dasatinib exhibited the highest potency to block PDGF-R signaling in orbital fibroblasts and may represent a promising compound for the treatment of GO as it was effective at low dosage and is associated with less side effects compared to imatinib mesylate and nilotinib. In this review the contribution of PDGF to the pathophysiology of GO as well as therapeutic approaches to target this PDGF-system will be addressed.

(c) 2014 European Thyroid Association Published by S. Karger AG, Basel

\section{Introduction}

Graves' disease (GD) is a common autoimmune disease that affects the thyroid gland. GD associated clinical features such as hyperactivity, heat intolerability, palpitations and weight loss are caused by excessive thyroid hormone secretion induced by thyroid-stimulating autoantibodies [1]. Apart from thyroid-related symptoms, GD patients can also develop extra-thyroidal manifestations such as ophthalmopathy and localized dermopathy $[1,2]$. Dermopathy is relatively rare and is present in approximately $4 \%$ of GD patients, while ophthalmopathy is clin-

\begin{tabular}{ll}
\hline KARGER & $\begin{array}{l}\text { @ 2014 European Thyroid Association } \\
\text { Published by S. Karger AG, Basel }\end{array}$ \\
$\begin{array}{l}\text { E-Mail karger@karger.com } \\
\text { www.karger.com/etj }\end{array}$ &
\end{tabular}

Willem A. Dik, PhD

Department of Immunology, Erasmus MC University Medical Center

NL-3000 CA Rotterdam (The Netherlands)

E-Mail w.dik@erasmusmc.nl 
ically manifest in approximately $25-50 \%$ of GD patients and strongly associated with smoking $[3,4]$.

Symptoms of Graves' ophthalmopathy (GO) are primarily of mechanical origin. An increase in orbital connective and/or muscle tissue within the space-limited bony orbit leads to protrusion of the eye and subsequent ocular symptoms, including upper eye lid retraction, edema, erythema of the periorbital tissues and conjunctivae, proptosis, strabismus, and blindness in severe cases [2]. Based on EUGOGO classification criteria the severity of GO can be categorized based on symptoms mentioned above into mild, moderate-to-severe and sight-threatening GO [5]. Mild GO cases are those having mild symptoms with minor influence on daily life and do not require treatment [5]. Moderate-to-severe cases are those who experience a major influence on daily life with higher degree of exophthalmos and diplopia, and require medical treatment [6]. Sight-threatening GO, including patients with dysthyroid optic neuropathy and/or corneal ulceration, requires immediate treatment $[4,5]$. Although other therapeutic approaches apart from corticosteroids, including biologicals targeting TNF- $\alpha$ or B-lymphocytes $[7,8]$ have been applied in GO, there is at current no convincing evidence for these treatment options and novel strategies are eagerly needed. This requires in depth insight into the complex pathophysiology of GO. In the past few years our group has shown that platelet-derived growth factor (PDGF), a major growth factor in health and disease, is elevated in orbital tissue from GO. In this review we will discuss the pathophysiology of GO and more specifically the role of PDGF herein, as well as potential therapeutic options to target the PDGF-system in GO.

\section{The Pathophysiology of GO}

The pathophysiology of GO comprises various cell types, inflammatory mediators and autoantibodies that interact with each other, resulting in orbital inflammation and tissue expansion. Immune cells involved in GO include at least T-lymphocytes, B-lymphocytes, monocytes, macrophages and mast cells, which activate orbital fibroblasts either via secreted factors or direct cell-cell contact $[9,10]$. Orbital fibroblast activation with resultant production of cytokines and glycosaminoglycans (especially hyaluronan), proliferation and adipocyte differentiation is considered to fulfill a central role in orbital tissue inflammation and expansion in GO (fig. 1).

Autoantibodies directed against the thyroid stimulating hormone receptor (TSHR) are considered as impor- tant contributors to GO and TSHR autoantibody titers correlate positively with disease severity and disease activity $[2,11]$. Orbital fibroblasts express TSHR, which increases when orbital fibroblasts differentiate into adipocytes $[12,13]$. TSHR stimulatory autoantibodies stimulate the production of cytokines, hyaluronan and adipocyte differentiation by orbital fibroblasts [14-18]. So far, however, hardly any information is available on mediators that regulate orbital TSHR expression, but IFN- $\gamma$, TGF- $\beta$ and TNF- $\alpha$ have been shown to downregulate TSHR expression on orbital fibroblasts, while this is enhanced by IL-6 $[19,20]$. It has been suggested that insulin-like growth factor-1 receptor (IGF-1R) stimulatory autoantibodies enhance the production of hyaluronan and the T cell chemoattractants IL-16 and CCL 5 by orbital fibroblasts and/or recruited fibrocytes [21-24]. These effects appear to depend on physical and functional interactions between the TSHR and IGF-1R, pointing at a functional interplay between TSHR autoantibodies, IGF-1R autoantibodies and their receptors in GO [25]. The contribution of stimulatory autoantibodies directed against IGF-1R in GO is however controversial [26] and therefore more studies are required to fully understand the role of IGF-1R and IGF-1R autoantibodies in the pathophysiology of GO.

The composition of the inflammatory cell infiltrate and inflammatory mediators within the orbital environment likely influences orbital fibroblast behaviour, and several different inflammatory mediators with orbital fibroblast activating capacities have been identified in orbital tissue from GO patients. Orbital inflammation in early GO is dominated by a T-helper 1 (Th1) cytokine environment with abundant production of cytokines such as IL-1 $\beta$, IL-2, IFN- $\gamma$ and TNF- $\alpha$ while in late GO a Th2 dominated cytokine environment with increased production of IL-4, IL-5 and IL-10 might be more prominent $[2,27-29]$. Of these it has been shown that IL-1 $\beta$ stimulates orbital fibroblasts to produce IL-6, IL-8, CCL2, CCL5 and IL-16, which are chemoattractants for B-lymphocytes, neutrophils, monocytes and T-lymphocytes $[30,31]$. Moreover, IL-1 $\beta$ strongly enhances hyaluronan production by orbital fibroblasts and stimulates orbital fibroblasts to differentiate into adipocytes [30, 32]. IFN- $\gamma$ induces HLA-DR, ICAM-1 and CD40 expression as well as CXCL9, CXCL10 and CXCL11 production by orbital fibroblasts from GO patients, indicating a role in lymphocyte recruitment and immune effector function [33-35]. In addition, IFN- $\gamma$ stimulates and acts synergistically with IL-1 $\beta$ on glycosaminoglycan synthesis by orbital fibroblasts $[36,37]$. In contrast to IL- $1 \beta$, IFN- $\gamma$ inhibits adipo- 


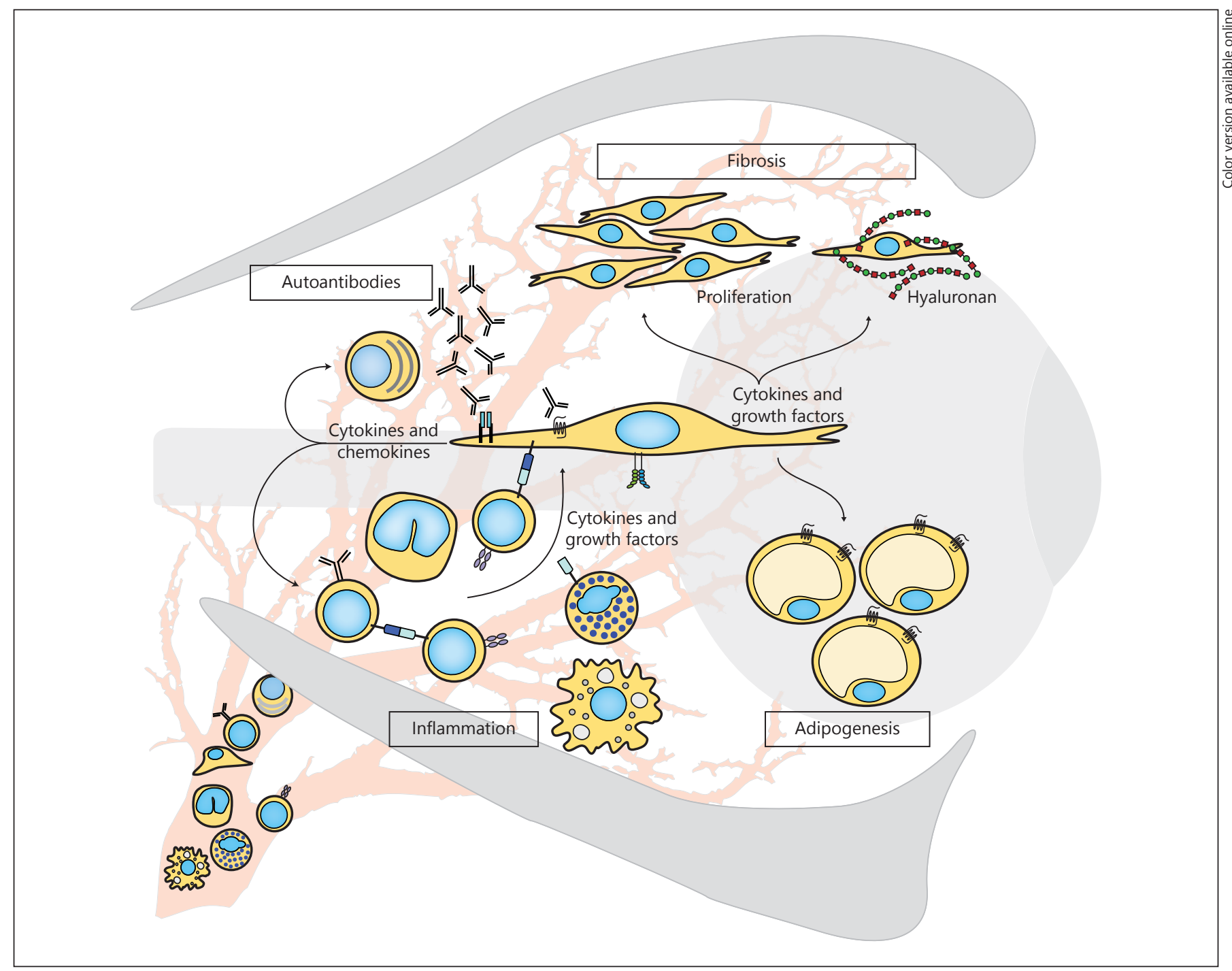

Fig. 1. Pathophysiology of GO. T-lymphocytes, B-lymphocytes, monocytes, macrophages and mast cells are recruited into the orbital tissue where they activate orbital fibroblasts via secreted factors or direct cell-cell contact to produce cytokines/chemokines and hyaluronan, to proliferate, and to differentiate into adipocytes.

genesis by orbital fibroblasts [19]. In the later stage of GO orbital fibroblasts proliferate and produce hyaluronan upon IL-4 stimulation $[37,38]$ while adipogenesis is not affected by IL-4 [20]. Interaction between CD154 (also called CD40-ligand) expressed by T-lymphocytes and CD40 expressed by orbital fibroblasts stimulates hyaluronan synthesis, ICAM-1 expression and production of various inflammatory mediators by orbital fibroblasts, amongst which IL-6 [39-42]. Next to directing B-lymphocyte effector functions, IL-6 may enhance adipogenesis by orbital fibroblasts in GO [43]. Besides the cyto-
Stimulatory autoantibodies directed against TSHR and IGF-1R can activate orbital fibroblasts to produce cytokines/chemokines, hyaluronan and to differentiate into adipocytes. Together these processes contribute to orbital tissue expansion in GO.

kines discussed above other factors with orbital fibroblast activating activities have been identified in GO and several of them, including their biologic effect on orbital fibroblasts, are given in table 1 .

In recent years numerous novel, targeted therapies have been introduced in clinical practice, like the biologicals and tyrosine kinase inhibitors (TKIs). Therapeutic strategies that target specific cytokines, growth factors or autoantibodies that display orbital fibroblast activating activity and are increased in $\mathrm{GO}$ may be promising. In the last few years our group has identified platelet-derived growth fac- 
Table 1. Factors that influence orbital fibroblast functions

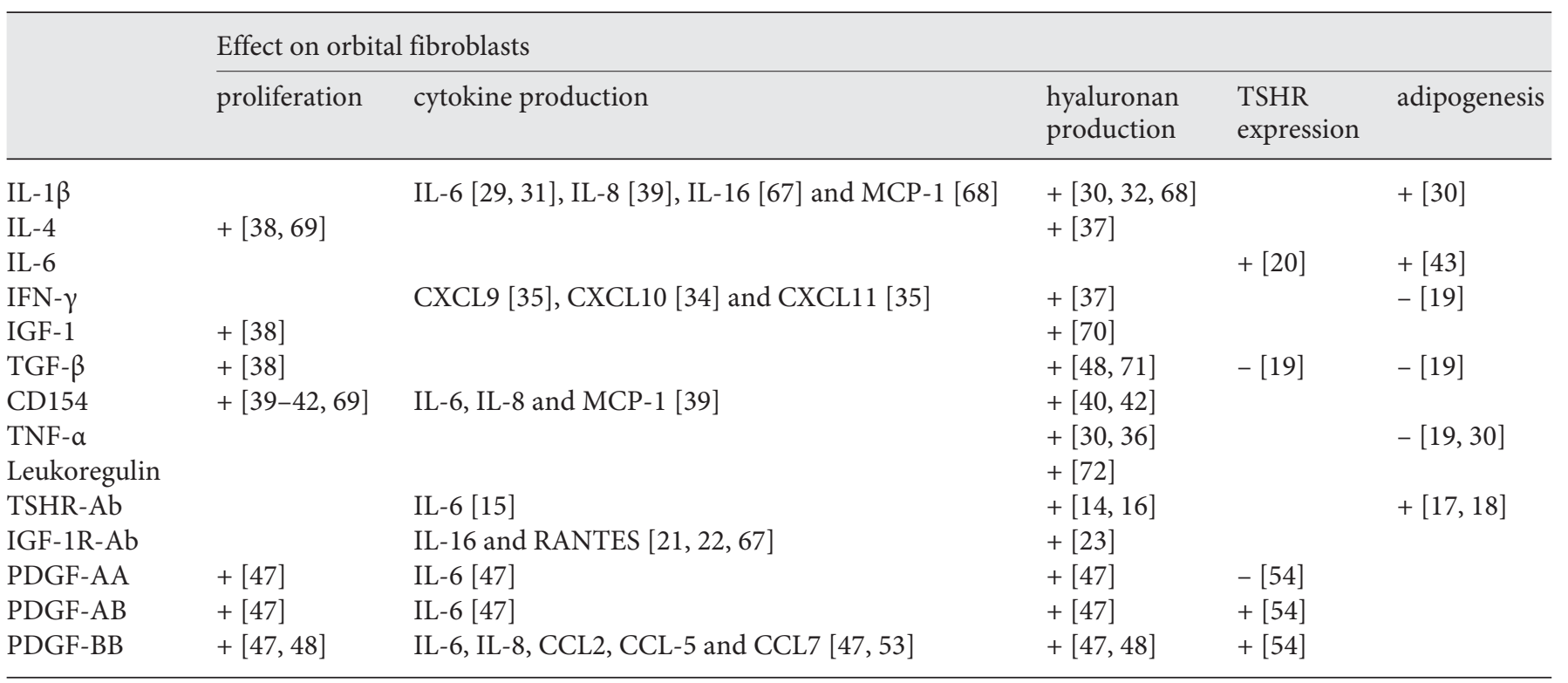

tor (PDGF) as a major contributor to the pathophysiology of GO and thus an attractive therapeutic target for this disease, which will be discussed in the next sections.

\section{Platelet-Derived Growth Factor}

PDGF is a family of growth stimulating polypeptides that exerts broad functions in health and disease [44]. There are four different PDGF genes that encode the peptide chains PDGF-A, PDGF-B, PDGF-C and PDGF-D [44]. Disulfide bridging between PDGF chains results in the formation of the homodimeric molecules PDGF-AA, PDGF-BB, PDGF-CC and PDGF-DD or the heterodimeric PDGF-AB molecule [44]. The pro-peptide chains of PDGF-A and PDGF-B dimerize intracellularly and have to be activated before secretion by removal of their $\mathrm{N}$-terminal ends [44]. PDGF-CC and PDGF-DD are secreted as latent molecules that contain CUB domains at their N-terminal ends [44]. Activation of these PDGF isoforms occurs after proteolytic removal of the CUB domains by proteases such as plasmin and tissue plasminogen activator [44].

PDGF dimers exert their biologic actions via activation of specific receptors consisting of two PDGF receptor (PDGF-R) chains ( $\alpha \alpha, \alpha \beta$ or $\beta \beta$ chains). The PDGF-A and PDGF-C chains are ligands for PDGF-R $\alpha$, the PDGF$\mathrm{D}$ chain is a ligand for PDGF-R $\beta$, while the PDGF-B chain can bind both to PDGF-R $\alpha$ and PDGF-R $\beta$, but with a higher affinity for PDGF-R $\beta$ [44]. PDGF-R chains consist of an extracellular and an intracellular part. The extracellular part contains five immunoglobulin-like domains while the intracellular part consists of split kinase domains (fig. 2a). Depending on the PDGF ligand that binds PDGF-R chains dimerize in either one of three dimeric forms; $\alpha \alpha, \alpha \beta$ or $\beta \beta$ (fig. 2a). The PDGF-receptor belongs to the tyrosine kinase receptor family and PDGF binding is followed by autophosphorylation of crucial tyrosine residues within the receptor chain (fig. $2 b$ ) with subsequent activation of downstream signaling molecules such as RAS-MAPK, PI3K and PLC $[44,45]$.

In normal physiology, PDGF signaling fulfills important roles in organogenesis, organ/tissue homeostasis and wound healing processes. For instance, PDGF-signaling is involved in alveogenesis, villus morphogenesis, spermatogenesis, nephrogenesis, angiogenesis, glomerulogenesis, tooth morphogenesis and development of dermis and lens [44]. Also in wound healing different PDGF isoforms play an important role as they recruit and activate neutrophils, macrophages and fibroblasts, thereby facilitating the tissue remodeling process [46]. However, sustained or elevated PDGF production and signaling is associated with many different diseases including cancers, vasculopathy and fibrosis [44]. A general characteristic of tissue fibrosis is excessive fibroblast activity with resultant hyperproliferation and extracellular matrix production by these cells, processes highly stimulated by PDGF isoforms and all contributing to GO as well. 


\section{Platelet-Derived Growth Factor in GO}

PDGF-A and PDGF-B chain expression levels were found to be increased by approximately $2-3$ fold in orbital tissue from GO patients, while expression levels of the PDGF-C, PDGF-D, PDGF-R $\alpha$ and PDGF-R $\beta$ chains were comparable to control orbital tissues $[47,48]$. Moreover, PDGF-A and PDGF-B chain expression was equally elevated in orbital tissue from GO patients with active or inactive disease, suggesting a role for the PDGF-AA, PDGF-AB and PDGF-BB isoforms throughout all GO disease stages. Monocytes, macrophages and mast cells, but not T-lymphocytes and B-lymphocytes, were identified as important sources of PDGF-A and PDGF-B chains in orbital tissues from GO patients [47]. Until now it is however unclear whether the orbital PDGF-production in $\mathrm{GO}$ is increased on a per-leukocyte basis or results from increased immune cell accumulation, but the more intense PDGF staining in mast cells in GO-orbital tissue compared to mast cells in control orbital tissue, as observed in our immunohistochemical studies, does support that increased production on a per cell basis contributes as well [47]. PDGF production is stimulated by a plethora of cytokines and growth factors, like IL-1 $\beta$, IFN- $\gamma$, TNF- $\alpha$ and TGF- $\beta$ [49-52], that are all increased in GO orbital tissue. However, so far factor(s) involved in driving PDGF production by different cell types in GO orbital tissue have not been examined.

PDGF-AA, PDGF-AB and PDGF-BB all stimulate the production of cytokines, amongst which IL-6, IL-8, CCL2 CCL5 and CCL7, by orbital fibroblasts derived from GO orbital tissue as well as control orbital tissue, with PDGFAA being the least potent and PDGF-BB the most potent inducer $[47,53]$. These cytokines have previously been implicated in the pathophysiology of GO and are able to attract and activate B-lymphocytes, T-lymphocytes, neutrophils, mast cells, monocytes and macrophages into the orbital tissue. Therefore PDGF isoforms control immune cell infiltration and activation in orbital tissue in GO through stimulation of cytokine/chemokine production by orbital fibroblasts.

TSHR autoantibody levels and orbital TSHR expression both positively correlate with GO disease activity and TSHR stimulating autoantibodies induce IL-6 and hyaluronan production by orbital fibroblasts, suggesting that TSHR stimulating autoantibodies contribute to development and severity of GO through orbital fibroblast activating properties [2]. Of the three PDGF-isoforms involved in $\mathrm{GO}$ it was found that PDGF-AB, but especially PDGF-BB, enhanced TSHR expression on orbital fibro-

PDGF in the Pathogenesis of GO and Target for Treatment blasts, while PDGF-AA did not affect TSHR expression [54]. The PDGF-induced upregulation of TSHR by orbital fibroblasts was associated with increased production of pro-inflammatory cytokines such as IL-6, IL-8, CCL2 and CCL 5 by orbital fibroblasts when stimulated with immunoglobulins obtained from Graves' disease (GD-IgG) patients. The GD-IgG effect was abrogated by c-AMP inhibition or a specific TSHR neutralizing antibody, indicating the TSHR-dependence [54]. These data underline the important role for PDGF-AB and PDGF-BB in amplifying the pathologic effects of TSHR stimulating autoantibodies in GO.

PDGF is well recognized for its role in wound healing and tissue fibrosis where it activates fibroblasts to proliferate and produce extracellular matrix components [44]. The orbital tissue expansion in early/active GO includes at least orbital fibroblast proliferation and hyaluronan production [2]. PDGF-AA, PDGF-AB and PDGF-BB all significantly stimulate orbital fibroblast proliferation, with PDGF-BB (inducing approximately $50 \%$ proliferation above control) being the most potent and PDGF-AA (inducing approximately $20 \%$ proliferation above control) being the least potent $[47,48]$. Also hyaluronan production by orbital fibroblasts is stimulated by all three PDGF-isoforms, again with the PDGF-B chain containing isoforms being the most potent [47, 48].

Altogether the data above demonstrate that PDGFisoforms, especially PDGF-AB and PDGF-BB, can be regarded as master regulators of several major pathophysiological process in GO. PDGF-AA, PDGF-AB and PDGF$\mathrm{BB}$ all stimulate proliferation, hyaluronan and cytokine/ chemokine production by orbital fibroblasts, while PDGF-AB and PDGF-BB also increase TSHR expression by orbital fibroblasts (fig. 3). As PDGF-A and PDGF-B chains are elevated in orbital tissue during all GO disease stages, PDGF-AA, AB and BB can be considered to represent true key factors in development and perpetuation of GO.

\section{Inhibition of PDGF Activity: An Attractive Possibility for the Treatment of Graves' Ophthalmopathy?}

Currently, the most effective well-tolerated treatment for active moderate-to-severe and sight-threatening GO is (high dose) corticosteroids, while radiotherapy or orbital decompression are considered when patients fail to respond to corticosteroids or for rehabilitating purposes $[4,5]$. Effectiveness of corticosteroid treatment relies mainly on the activity of the disease, with a high success 


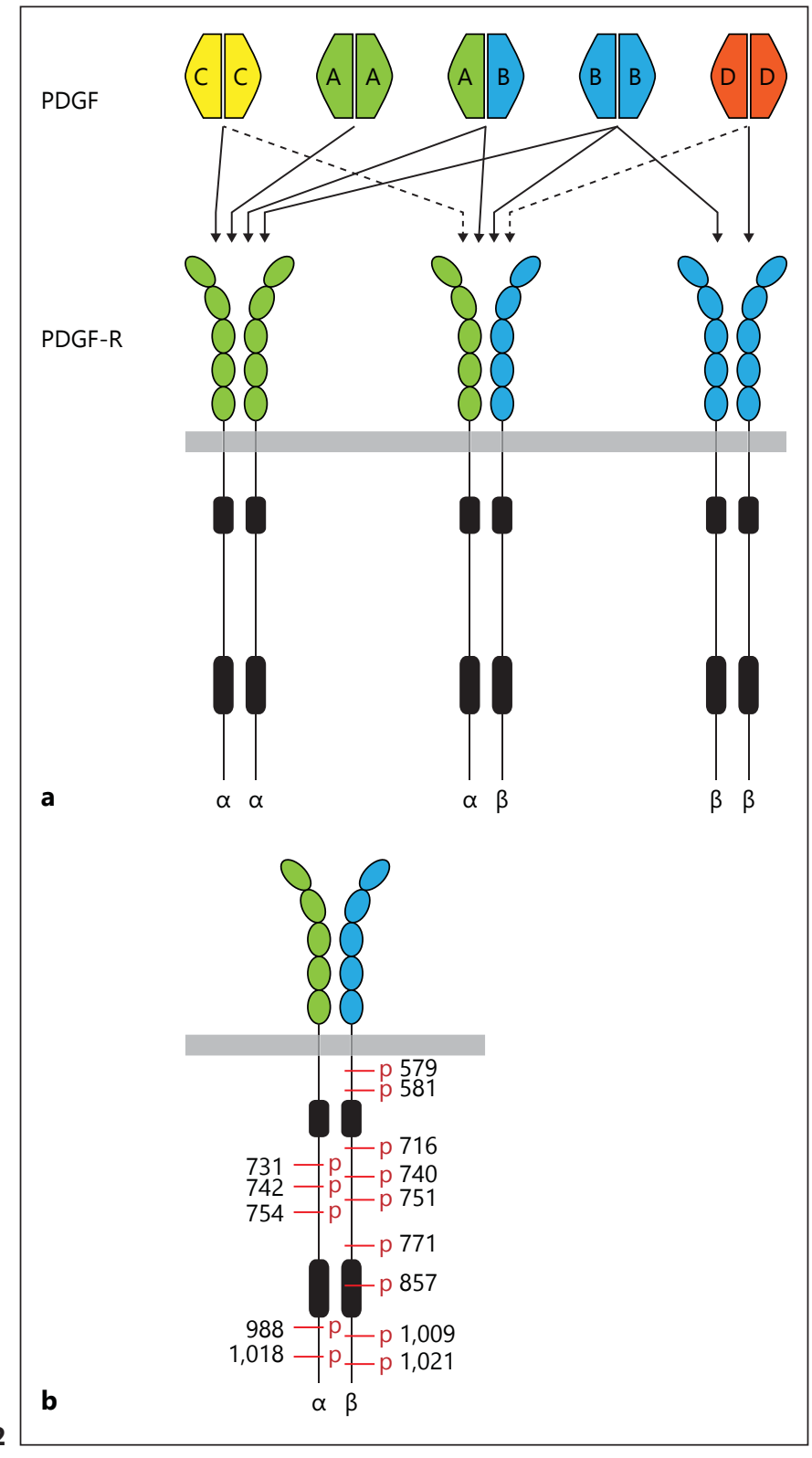

Fig. 2. PDGF and PDGF-receptor. a PDGF-A and PDGF-C chains are ligands for PDGF-R $\alpha, P D G F-D$ chain is a ligand for PDGF-R $\beta$, while the PDGF-B chain can bind both to PDGF-R $\alpha$ and PDGF$\mathrm{R} \beta$. Dotted lines indicate weak interactions or conflicting results [44]. b Autophosphorylation of crucial tyrosine residues within the receptor chain results in the activation of downstream signaling molecules.

Fig. 3. Role of PDGF signaling in GO. Monocytes, macrophages and mast cells produce PDGF-A and PDGF-B chains in orbital tissue from $\mathrm{GO}$, resulting in the formation of PDGF-AA, PDGF-AB and PDGF-BB dimeric molecules. These PDGF-isoforms stimulate proliferation, cytokine and hyaluronan production by orbital fibroblasts while PDGF-AB and PDGF-BB also enhance TSHR expression on orbital fibroblasts. In general PDGF-BB is the PDGFisoform exhibiting the most potent effect on orbital fibroblasts, while PDGF-AA is the weakest.
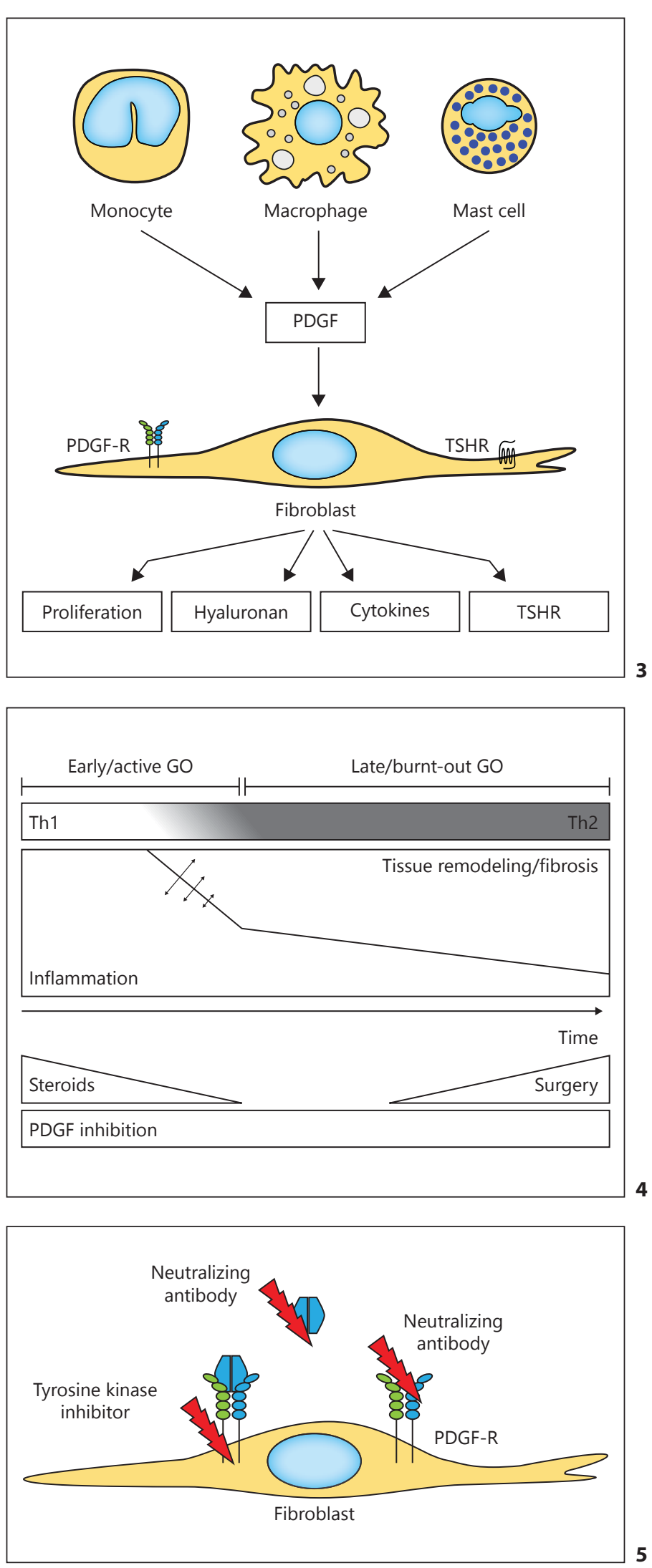

(For legends 4 and 5 see next page.) 
rate when introduced in the initial active inflammatory phase of the disease $[4,5]$. However, corticosteroid treatment may negatively influence the tissue remodeling or fibrotic phase when inflammation has subsided [5]. Corticosteroids, such as dexamethasone, stimulate PDGF-B production by macrophages and enhance PDGF-Ra expression on fibroblasts, which augments fibroblast effector functions in lung fibrosis [55-57]. In contrast to the ambivalent effects that corticosteroids can have with regard to inflammation, tissue remodeling and fibrosis, the ideal therapy for GO should be effective regardless of the stage of disease. However, so far novel medical treatment options for GO have mainly concentrated on therapeutics directed at immune cells (e.g. B-lymphocytes) or mediators (e.g. TNF- $\alpha$ ) that mainly are involved in the active inflammatory phase of GO $[7,8]$.

PDGF targeting seems an attractive therapeutic option in GO, as PDGF-driven orbital fibroblast activation most likely occurs in all stages of GO (fig. 4). Several approaches to interfere with PDGF-signaling in GO can be thought of: (1) neutralization of PDGF-molecules, for instance with specific neutralizing antibodies or soluble receptor molecules, (2) blockage of the PDGF-receptor chains with neutralizing antibodies or dominant negative ligands and (3) inhibition of PDGF-receptor signaling by using tyrosine-kinase inhibitors that prevent receptor autophosphorylation upon ligand binding (fig. 5) [44].

Although neutralizing antibodies directed towards PDGF isoforms or PDGF-receptors are currently not available for clinical use, such an approach might be of

Fig. 4. A hypothetical scheme of the pathophysiology and treatment of GO. Early GO is characterized by a Th1-dominated inflammatory environment which leads to massive orbital tissue inflammation and edema. In time, this Th1 environment is skewed towards a Th2-dominated environment in which inflammation subsides, but fibrotic tissue remodeling continues. Current mainstream treatment of GO consists of corticosteroids and surgery, of which the corticosteroids have a relatively high success rate when introduced in the active inflammatory phase of the disease, but may negatively influence the tissue remodeling or fibrotic phase when inflammation has subsided. Surgery may be effective in early/active stages of $\mathrm{GO}$, but is predominantly used for rehabilitation of GO patients. Increased PDGF activity contributes to all stages of GO and inhibition of PDGF activity may therefore be effective in all stages of GO. Fig. 5. Approaches to target PDGF signaling. The PDGF system can be blocked by targeting the PDGF molecule, for instance with a neutralizing antibody, or by targeting the PDGF-Receptor, for instance with a neutralizing antibody or a tyrosine kinase inhibitor with specificity for the PDGF-Receptor.

PDGF in the Pathogenesis of GO and Target for Treatment benefit in the treatment of GO as we found that a neutralizing antibody directed towards PDGF-BB reduced IL-6 and hyaluronan secretion by orbital tissue from GO patients in a newly developed orbital tissue culture approach [47]. Remarkably, in this culture system inhibition of PDGF-AA with a neutralizing antibody was hardly effective, once more underlining the importance of PDGF-B chain containing PDGF isoforms in the pathophysiology of GO.

Several tyrosine kinase inhibitors (TKIs) that exhibit specificity for the PDGF-receptor, amongst which imatinib mesylate and nilotinib, are widely applied to treat BCR-ABL positive chronic myeloid leukemia (CML) as the tyrosine kinase ABL is a target for these TKIs as well [58]. In addition, imatinib mesylate has been used successfully to treat gastrointestinal tumors and mastocytosis by targeting c-Kit kinase activity $[59,60]$. We found, that imatinib mesylate and nilotinib both were able to prevent PDGF-induced TSHR expression, proliferation, cytokine and hyaluronan production by orbital fibroblasts from GO patients [47, 48, 53, 54]. Moreover, imatinib mesylate attenuated IL- 6 and hyaluronan secretion by cultured GO orbital tissue, while the TNF- $\alpha$ neutralizing agent adalimumab only reduced IL- 6 secretion [61]. Although these data point at the attractiveness of TKI usage in the treatment of GO, imatinib mesylate and nilotinib were found to cause serious side effects such as peri-orbital edema, peripheral arterial occlusive disease and cerebrovascular events in CML treatment [62]. Moreover, it was recently shown that imatinib mesylate can stimulate adipogenesis by orbital fibroblasts [63], although this was at high imatinib mesylate concentration. Based on the described adverse effects, imatinib mesylate and nilotinib are not directly regarded as candidate TKIs for a clinical study in GO, at least not when applied in the same dose as used for CML treatment.

Recently, we found that dasatinib, a second generation TKI with FDA approval for CML treatment, effectively inhibits PDGF-BB-induced proliferation, cytokine (CCL2, IL-6, IL-8) and hyaluronan production by orbital fibroblasts as well as cytokine and hyaluronan production by whole orbital tissue from active GO [64]. Although dasatinib is a more promiscuous TKI than imatinib mesylate and nilotinib, it displays the highest inhibitory potency (pIC50) for PDGF-R $\alpha$ and PDGF-R $\beta$ amongst all TKIs approved for clinical use [65]. In line with this, dasatinib prevented PDGF-induced orbital fibroblast activation at concentrations far lower than that required for imatinib mesylate [64]. Dasatinib has been found to stim- 
ulate adipogenesis by human bone marrow-derived mesenchymal stromal cells but not human skin fibroblasts [66]. This implies that an adipogenic effect of dasatinib may be cell type specific, which warrants studies with orbital fibroblasts as well. Although dasatinib is well tolerated some side effects like pleural effusion, skin rash, vomiting, diarrhea, fatigue, headache, anemia, thrombocytopenia and neutropenia have been described [64]. Occurrence of side effects cannot be excluded when dasatinib is used for GO treatment, but the incidences reported in cancer treatment are relatively low, and the occasional occurrence of pleural effusion was reversible upon discontinuation of the therapy. Moreover, the data available so far suggest that dasatinib might be effective in reducing orbital tissue inflammation and expansion at relatively low dose, which may limit the occurrence of side effects as well. Nevertheless, it should be taken into account that GO may show a self-limiting course. Therefore, the use of a compound like dasatinib, with potential side effects, should be introduced in clinics with the greatest precaution.
In summary, PDGF molecules fulfill a central role in the pathophysiology of GO by activation of orbital fibroblasts. Therefore, targeting of the PDGF system should be regarded as therapeutic option for GO.

\section{Acknowledgement}

The research for this manuscript was (in part) performed within the framework of the Erasmus Postgraduate School Molecular Medicine. The authors thank Sandra de Bruin - Versteeg for her assistance with the figures.

\section{Funding Statement}

This research received no specific grant from any funding agency in the public, commercial or not-for-profit sectors.

\section{Disclosure Statement}

None.

\section{References}

1 Weetman AP: Graves' disease. N Engl J Med 2000;343:1236-1248.

-2 Bahn RS: Graves' ophthalmopathy. N Engl J Med 2010;362:726-738.

-3 Tanda ML, Piantanida E, Liparulo L, et al: Prevalence and natural history of Graves' orbitopathy in a large series of patients with newly diagnosed graves' hyperthyroidism seen at a single center. J Clin Endocrinol Metab 2013;98:1443-1449.

4 Marcocci C, Marino M: Treatment of mild, moderate-to-severe and very severe Graves' orbitopathy. Best Pract Res Clin Endocrinol Metab 2012;26:325-337.

5 Bartalena L, Baldeschi L, Dickinson A, et al: Consensus statement of the European Group on Graves' orbitopathy (EUGOGO) on management of GO. Eur J Endocrinol 2008; 158: 273-285.

6 Kahaly GJ, Pitz S, Hommel G, et al: Randomized, single blind trial of intravenous versus oral steroid monotherapy in Graves' orbitopathy. J Clin Endocrinol Metab 2005;90:52345240.

7 Salvi M, Vannucchi G, Beck-Peccoz P: Potential utility of rituximab for Graves' orbitopathy. J Clin Endocrinol Metab 2013;98:4291-4299.

-8 Paridaens D, van den Bosch WA, van der Loos TL, et al: The effect of etanercept on Graves' ophthalmopathy: a pilot study. Eye (Lond) 2005;19:1286-1289.

9 Weetman AP, Cohen S, Gatter KC, et al: Immunohistochemical analysis of the retrobul- bar tissues in Graves' ophthalmopathy. Clin Exp Immunol 1989;75:222-227.

10 Pappa A, Calder V, Ajjan R, et al: Analysis of extraocular muscle-infiltrating $\mathrm{T}$ cells in thyroid-associated ophthalmopathy (TAO). Clin Exp Immunol 1997;109:362-369.

11 Gerding MN, van der Meer JWC, Broenink $\mathrm{M}$, et al: Association of thyrotrophin receptor antibodies with the clinical features of Graves' ophthalmopathy. Clin Endocrinol 2000;52: 267-271.

12 Valyasevi RW, Harteneck DA, Dutton CM, et al: Stimulation of adipogenesis, peroxisome proliferator-activated receptor-gamma (PPARgamma), and thyrotropin receptor by PPARgamma agonist in human orbital preadipocyte fibroblasts. J Clin Endocrinol Metab 2002;87:2352-2358.

13 Valyasevi RW, Erickson DZ, Harteneck DA, et al: Differentiation of human orbital preadipocyte fibroblasts induces expression of functional thyrotropin receptor. J Clin Endocrinol Metab 1999;84:2557-2562.

14 van Zeijl CJ, Fliers E, van Koppen CJ, et al: Thyrotropin receptor-stimulating Graves' disease immunoglobulins induce hyaluronan synthesis by differentiated orbital fibroblasts from patients with Graves' ophthalmopathy not only via cyclic adenosine monophosphate signaling pathways. Thyroid 2011;21:169176.

15 Kumar S, Schiefer R, Coenen MJ, et al: A stimulatory thyrotropin receptor antibody (M22) and thyrotropin increase interleukin-6 expression and secretion in Graves' orbital preadipocyte fibroblasts. Thyroid 2010;20:5965.

16 Zhang L, Bowen T, Grennan-Jones F, et al: Thyrotropin receptor activation increases hyaluronan production in preadipocyte fibroblasts: contributory role in hyaluronan accumulation in thyroid dysfunction. J Biol Chem 2009;284:26447-26455.

17 Kumar S, Nadeem S, Stan MN, et al: A stimulatory TSH receptor antibody enhances adipogenesis via phosphoinositide 3-kinase activation in orbital preadipocytes from patients with Graves' ophthalmopathy. J Mol Endocrinol 2011;46:155-163.

18 Zhang L, Baker G, Janus D, et al: Biological effects of thyrotropin receptor activation on human orbital preadipocytes. Invest Ophthalmol Vis Sci 2006;47:5197-5203.

19 Valyasevi RW, Jyonouchi SC, Dutton CM, et al: Effect of tumor necrosis factor-alpha, interferon-gamma, and transforming growth factor-beta on adipogenesis and expression of thyrotropin receptor in human orbital preadipocyte fibroblasts. J Clin Endocrinol Metab 2001;86:903-908.

20 Jyonouchi SC, Valyasevi RW, Harteneck DA, et al: Interleukin-6 stimulates thyrotropin receptor expression in human orbital preadipocyte fibroblasts from patients with Graves' ophthalmopathy. Thyroid 2001;11: 929-934. 
21 Pritchard J, Han R, Horst N, et al: Immunoglobulin activation of $\mathrm{T}$ cell chemoattractant expression in fibroblasts from patients with Graves' disease is mediated through the insulin-like growth factor I receptor pathway. J Immunol 2003;170:6348-6354.

-22 Smith TJ, Hegedus L, Douglas RS: Role of insulin-like growth factor-1 (IGF-1) pathway in the pathogenesis of Graves' orbitopathy. Best Pract Res Clin Endocrinol Metab 2012;26: 291-302.

23 Smith TJ, Hoa N: Immunoglobulins from patients with Graves' disease induce hyaluronan synthesis in their orbital fibroblasts through the self-antigen, insulin-like growth factor-I receptor. J Clin Endocrinol Metab 2004;89: 5076-5080.

24 Douglas RS, Afifiyan NF, Hwang CJ, et al: Increased generation of fibrocytes in thyroidassociated ophthalmopathy. J Clin Endocrinol Metab 2010;95:430-438.

25 Tsui S, Naik V, Hoa N, et al: Evidence for an association between thyroid-stimulating hormone and insulin-like growth factor 1 receptors: a tale of two antigens implicated in Graves' disease. J Immunol 2008;181:43974405.

26 Minich WB, Dehina N, Welsink T, et al: Autoantibodies to the IGF1 receptor in Graves' orbitopathy. J Clin Endocrinol Metab 2013; 98:752-760.

27 Wakelkamp IM, Bakker O, Baldeschi L, et al TSH-R expression and cytokine profile in orbital tissue of active versus inactive Graves' ophthalmopathy patients. Clin Endocrinol (Oxf) 2003;58:280-287.

28 Aniszewski JP, Valyasevi RW, Bahn RS: Relationship between disease duration and predominant orbital T cell subset in Graves' ophthalmopathy. J Clin Endocrinol Metab 2000; 85:776-780.

29 Forster G, Otto E, Hansen C, et al: Analysis of orbital T cells in thyroid-associated ophthalmopathy. Clin Exp Immunol 1998;112:427434.

- 30 Cawood TJ, Moriarty P, O’Farrelly C, et al: The effects of tumour necrosis factor-alpha and interleukin 1 on an in vitro model of thyroid-associated ophthalmopathy; contrasting effects on adipogenesis. Eur J Endocrinol 2006;155:395-403.

- 31 Chen B, Tsui S, Smith TJ: IL-1 beta induces IL-6 expression in human orbital fibroblasts: identification of an anatomic-site specific phenotypic attribute relevant to thyroid-associated ophthalmopathy. J Immunol 2005;175: 1310-1319.

- 32 Kaback LA, Smith TJ: Expression of hyaluronan synthase messenger ribonucleic acids and their induction by interleukin-1beta in human orbital fibroblasts: potential insight into the molecular pathogenesis of thyroid-associated ophthalmopathy. J Clin Endocrinol Metab 1999;84:4079-4084.

-33 Yan SX, Wang Y: Inhibitory effects of Triptolide on interferon-gamma-induced human leucocyte antigen-DR, intercellular adhesion molecule-1, CD40 expression on retro-ocular fibroblasts derived from patients with Graves' ophthalmopathy. Clin Experiment Ophthalmol 2006;34:265-271.

34 Antonelli A, Rotondi M, Ferrari SM, et al: Interferon-gamma-inducible alpha-chemokine CXCL10 involvement in Graves' ophthalmopathy: modulation by peroxisome proliferator-activated receptor-gamma agonists. J Clin Endocrinol Metab 2006;91:614-620.

35 Antonelli A, Ferrari SM, Fallahi P, et al: Monokine induced by interferon gamma (IFNgamma) (CXCL9) and IFNgamma inducible T-cell alpha-chemoattractant (CXCL11) involvement in Graves' disease and ophthalmopathy: modulation by peroxisome proliferator-activated receptorgamma agonists. J Clin Endocrinol Metab 2009;94:1803-1809.

- 36 Metcalfe RA, Weetman AP: Stimulation of extraocular muscle fibroblasts by cytokines and hypoxia: possible role in thyroid-associated ophthalmopathy. Clin Endocrinol (Oxf) 1994;40:67-72.

37 Han R, Smith TJ: T helper type 1 and type 2 cytokines exert divergent influence on the induction of prostaglandin E2 and hyaluronan synthesis by interleukin-1beta in orbital fibroblasts: implications for the pathogenesis of thyroid-associated ophthalmopathy. Endocrinology 2006;147:13-19.

38 Heufelder AE, Bahn RS: Modulation of Graves' orbital fibroblast proliferation by cytokines and glucocorticoid receptor agonists. Invest Ophthalmol Vis Sci 1994;35:120-127.

39 Hwang CJ, Afifiyan N, Sand D, et al: Orbital fibroblasts from patients with thyroid-associated ophthalmopathy overexpress CD40: CD154 hyperinduces IL-6, IL-8, and MCP-1. Invest Ophthalmol Vis Sci 2009;50:22622268.

40 Cao HJ, Wang HS, Zhang Y, et al: Activation of human orbital fibroblasts through CD40 engagement results in a dramatic induction of hyaluronan synthesis and prostaglandin endoperoxide $\mathrm{H}$ synthase-2 expression. Insights into potential pathogenic mechanisms of thyroid-associated ophthalmopathy. J Biol Chem 1998;273:29615-29625.

-41 Sempowski GD, Rozenblit J, Smith TJ, et al: Human orbital fibroblasts are activated through CD40 to induce proinflammatory cytokine production. Am J Physiol 1998;274: C707-C714.

42 Zhao LQ, Wei RL, Cheng JW, et al: The expression of intercellular adhesion molecule-1 induced by CD40-CD40L ligand signaling in orbital fibroblasts in patients with Graves' ophthalmopathy. Invest Ophthalmol Vis Sci 2010;51:4652-4660.

43 Bahn RS: Thyrotropin receptor expression in orbital adipose/connective tissues from patients with thyroid-associated ophthalmopathy. Thyroid 2002;12:193-195.

44 Andrae J, Gallini R, Betsholtz C: Role of platelet-derived growth factors in physiology and medicine. Genes Dev 2008;22:1276-1312.
45 Claesson-Welsh L: Platelet-derived growth factor receptor signals. J Biol Chem 1994;269: 32023-32026.

-46 Pierce GF, Mustoe TA, Altrock BW, et al: Role of Platelet-Derived Growth-Factor in Wound-Healing. Journal of Cellular Biochemistry 1991;45:319-326.

47 van Steensel L, Paridaens D, van Meurs M, et al: Orbit-infiltrating mast cells, monocytes, and macrophages produce PDGF isoforms that orchestrate orbital fibroblast activation in Graves' ophthalmopathy. J Clin Endocrinol Metab 2012;97:E400-E408.

48 van Steensel L, Paridaens D, Schrijver B, et al: Imatinib mesylate and AMN107 inhibit PDGF-signaling in orbital fibroblasts: a potential treatment for Graves' ophthalmopathy. Invest Ophthalmol Vis Sci 2009;50:3091-3098.

49 Raines EW, Dower SK, Ross R: Interleukin-1 mitogenic activity for fibroblasts and smooth muscle cells is due to PDGF-AA. Science 1989;243:393-396.

50 Suzuki H, Shibano K, Okane M, et al: Interferon-gamma modulates messenger RNA levels of c-sis (PDGF-B chain), PDGF-A chain, and IL-1 beta genes in human vascular endothelial cells. Am J Pathol 1989;134:35-43.

51 Liu JY, Brass DM, Hoyle GW, et al: TNF-alpha receptor knockout mice are protected from the fibroproliferative effects of inhaled asbestos fibers. Am J Pathol 1998;153:18391847.

52 Daniel TO, Gibbs VC, Milfay DF, et al: Agents that increase cAMP accumulation block endothelial c-sis induction by thrombin and transforming growth factor-beta. J Biol Chem 1987;262:11893-11896.

53 van Steensel L, Paridaens D, Dingjan GM, et al: Platelet-derived growth factor-BB: a stimulus for cytokine production by orbital fibroblasts in Graves' ophthalmopathy. Invest Ophthalmol Vis Sci 2010;51:1002-1007.

54 van Steensel L, Hooijkaas H, Paridaens D, et al: PDGF enhances orbital fibroblast responses to TSHR stimulating autoantibodies in Graves' ophthalmopathy patients. J Clin Endocrinol Metab 2012;97:E944-E953.

55 Dik WA, Versnel MA, Naber BA, et al: Dexamethasone treatment does not inhibit fibroproliferation in chronic lung disease of prematurity. Eur Respir J 2003;21:842-847.

56 Warshamana GS, Martinez S, Lasky JA, et al: Dexamethasone activates expression of the PDGF-alpha receptor and induces lung fibroblast proliferation. Am J Physiol 1998;274: L499-L507.

57 Haynes AR, Shaw RJ: Dexamethasone-induced increase in platelet-derived growth factor (B) mRNA in human alveolar macrophages and myelomonocytic HL60 macrophage-like cells. Am J Respir Cell Mol Biol 1992;7:198-206.

58 Steegmann JL, Cervantes F, le Coutre P, et al: Off-target effects of BCR-ABL1 inhibitors and their potential long-term implications in patients with chronic myeloid leukemia. Leuk Lymphoma 2012;53:2351-2361.
PDGF in the Pathogenesis of GO and Target for Treatment
Eur Thyroid J 2014;3:217-226 DOI: $10.1159 / 000367968$ 
59 Demetri GD, von Mehren M, Blanke CD, et al: Efficacy and safety of imatinib mesylate in advanced gastrointestinal stromal tumors. N Engl J Med 2002;347:472-480.

60 Droogendijk HJ, Kluin-Nelemans HJ, van Doormaal JJ, et al: Imatinib mesylate in the treatment of systemic mastocytosis: a phase II trial. Cancer 2006;107:345-351.

61 van Steensel L, van Hagen PM, Paridaens D, et al: Whole orbital tissue culture identifies imatinib mesylate and adalimumab as potential therapeutics for Graves' ophthalmopathy. Br J Ophthalmol 2011;95:735-738.

-62 Kim TD, Rea D, Schwarz M, et al: Peripheral artery occlusive disease in chronic phase chronic myeloid leukemia patients treated with nilotinib or imatinib. Leukemia 2013;27: 1316-1321.

63 Li H, Fitchett C, Kozdon K, et al: Independent adipogenic and contractile properties of fibroblasts in graves' orbitopathy: an in vitro model for the evaluation of treatments. PLoS One 2014;9:e95586.
64 Virakul S, Dalm VA, Paridaens D, et al: The tyrosine kinase inhibitor dasatinib effectively blocks PDGF-induced orbital fibroblast activation. Graefes Arch Clin Exp Ophthalmol 2014;252:1101-1109.

65 Kitagawa D, Yokota K, Gouda M, et al: Activity-based kinase profiling of approved tyrosine kinase inhibitors. Genes Cells 2013;18: 110-122.

66 Borriello A, Caldarelli I, Basile MA, et al: The tyrosine kinase inhibitor dasatinib induces a marked adipogenic differentiation of human multipotent mesenchymal stromal cells. PLoS One 2011;6:e28555.

67 Pritchard J, Horst N, Cruikshank W, et al: Igs from patients with Graves' disease induce the expression of $\mathrm{T}$ cell chemoattractants in their fibroblasts. J Immunol 2002;168:942-950.
68 Paik JS, Cho WK, Oh EH, et al: Palmitate induced secretion of IL- 6 and MCP-1 in orbital fibroblasts derived from patients with thyroid-associated ophthalmopathy. Mol Vis 2012;18:1467-1477.

69 Feldon SE, Park DJ, O’Loughlin CW, et al: Autologous T-lymphocytes stimulate proliferation of orbital fibroblasts derived from patients with Graves' ophthalmopathy. Invest Ophthalmol Vis Sci 2005;46:3913-3921.

70 Imai Y, Odajima R, Inoue Y, et al: Effect of growth factors on hyaluronan and proteoglycan synthesis by retroocular tissue fibroblasts of Graves' ophthalmopathy in culture. Acta Endocrinol (Copenh) 1992;126:541-552.

71 Imai Y, Ibaraki K, Odajima R, et al: Analysis of proteoglycan synthesis by retro-ocular tissue fibroblasts under the influence of interleukin 1 beta and transforming growth factorbeta. Eur J Endocrinol 1994;131:630-638.

72 Smith TJ, Wang HS, Evans CH: Leukoregulin is a potent inducer of hyaluronan synthesis in cultured human orbital fibroblasts. Am J Physiol 1995;268:C382-C388. 